\title{
Ciclo de vida de Culex quinquefasciatus Say, 1826 (Diptera: Culicidae) bajo condiciones no controladas en Bogotá
}

\author{
Myriam Janeth Salazar, Ligia Inés Moncada \\ Facultad de Medicina, Universidad Nacional de Colombia, Bogotá, D.C., Colombia.
}

El objetivo de este trabajo fue estudiar varios aspectos del crecimiento y el desarrollo de los estadios inmaduros de Culex quinquefasciatus Say, 1826 (Diptera: Culicidae), especie antropofílica frecuentemente encontrada en Bogotá. Con este fin, se realizaron dos experimentos en diferentes épocas del año 2001 (enero-febrero y septiembre-octubre), bajo condiciones no controladas (luz, temperatura y humedad relativa). Se colocaron recipientes plásticos transparentes con agua de charca a la que se le adicionó concentrado para perro; se tomaron cuatro balsas al azar para estudiar el ciclo de vida utilizando los parámetros de la tabla de vida: mortalidad y supervivencia. Las hembras ovipositaron entre cinco y ocho días después de la ingestión de sangre. El número de huevos por balsa varió entre 152 y 203. La eclosión de larvas L1 fue de $50 \%$ en el primer experimento y de $75 \%$ en el segundo. Se destacó la naturaleza no sincrónica de la eclosión de las L1, la menor duración proporcional del estadio de pupa (11\% del tiempo del desarrollo total) y la eficiencia del cambio pupa-adulto $(98,61 \%)$. Se reporta una menor duración del ciclo de lo informado previamente. Además, los altos porcentajes de eclosión $(83,58 \%)$, pupación $(86,63 \%)$ y emergencia $(98,61 \%)$ con las condiciones presentes para estos experimentos (temperatura media $14,8^{\circ} \mathrm{C}$ y $15,1^{\circ} \mathrm{C}$ y humedad relativa del $72,5 \%$ y $74,1 \%$, respectivamente) indican el alto grado de adaptación de C. quinquefasciatus al ambiente bogotano. Estas características, más la capacidad vectorial y la resistencia a los insecticidas, hacen de esta especie un problema de salud pública.

Palabras clave: Culex quinquefasciatus/crecimiento\&desarrollo, mortalidad.

Life cycle of Culex quinquefasciatus Say (Diptera: Culicidae) under uncontrolled conditions

Aspects of the growth and development were described for immature stages of Culex quinquefasciatus Say, 1826 (Diptera: Culicidae), an antropophilic mosquito species found frequently in Bogotá, Colombia. Two experiments were carried out during January-February and September-October of 2001 under ambient environmental conditions. Oviposition occured 5-8 days after blood ingestion. Females laid eggs in plastic containers filled with pooled water with high organic material content. The number of eggs per raft varied between 152 and 203. For the 2 trials, the hatch rate was $50 \%$ and $75 \%$. The asynchronous egg hatch, the short duration of the pupal stage $(11 \%$ of the total development time, and the high efficiency of adult emergence from the pupal stage (98.6\%) were noted. In general, these development times were shorter compared to those reported by other authors. Moreover, the high percentages of hatch $(83.6 \%)$, pupation $(86.6 \%)$ and emergence $(98.6 \%)$ under the average temperature conditions of $14.8^{\circ} \mathrm{C}$ and $15.1^{\circ} \mathrm{C}$, and average relative humidity of $72.5 \%$ and $74.1 \%$, respectively) demonstrated adaptation of $C$. quinquefasciatus to Bogotá's cool, high altitude environment. These characteristics, together with its vectorial capacity and the resistance to chemical control methods, could make this species become a risk for the health of human populations.

Key words: Culex quinquefasciatus/growth\&development, mortality. 
Los mosquitos son los artrópodos hematófagos más comunes y de mayor importancia médica y veterinaria porque causan molestias y transmiten agentes productores de enfermedades a gran variedad de especies de aves y mamíferos, incluso los seres humanos. Hasta el momento se han descrito cerca de 3.000 especies de mosquitos $y$, aunque las especies vectoras constituyen una baja proporción, los problemas ocasionados por las enfermedades producidas por los agentes transmitidos por estos insectos son serios (1).

Los mosquitos del grupo Culex pipiens Linnaeus, 1758 (Diptera: Culicidae) son vectores de algunos arbovirus y nemátodos que afectan al hombre y los animales (2-4). Los miembros de este grupo se distribuyen a lo largo y ancho del planeta, principalmente con dos especies: Culex pipiens, presente en zonas templadas y Culex quinquefasciatus, Say, 1823, que habita en regiones tropicales y subtropicales y abarca hasta las isotermas de $20^{\circ} \mathrm{C}(5)$; abunda principalmente en América y África tropical, Medio y Lejano Oriente, sur de Asia, Nueva Guinea, Australia y el sur de Estados Unidos, aunque existen zonas de intergradación (Norteamérica, norte del Japón, suroriente de Australia, Medio Oriente, área central de Argentina, entre los $30^{\circ}$ y los $33^{\circ}$ de latitud sur, y África) donde se han reportado híbridos (67). Las dos especies se diferencian por caracteres morfológicos y morfométricos, y también presentan comportamientos y características fisiológicas diferentes (7-11).

La posición taxonómica de ambas especies permanece aún indefinida en las áreas geográficas donde sus rangos de distribución se superponen; para distinguirlas se han utilizado características de tipo morfométrico (relación dv/d; dv: distancia entre las extremidades de los brazos dorsales y ventrales del mesosoma y d, distancia entre las extremidades de los brazos dorsales del

Correspondencia:

Ligia Inés Moncada, Oficina 314F, Laboratorio de Parasitología, Facultad de Medicina, Universidad Nacional, Ciudad Universitaria, Bogotá, D.C., Colombia.

Teléfono: (571) 3165505 y (571) 3165000 , extensión 15032; fax: (571) 3165405

limoncadaa@unal.edu.co

Recibido: 22/06/04; aceptado: 13/09/04 mesosoma; de la genitalia masculina), fisiológico (mecanismos de hibernación y diapausa, desarrollo de etapas inmaduras) etológico (búsqueda de hospedero) y ecológico (distribución geográfica) (7). Según Forattini $(1965)(10,11)$, las dos especies se pueden diferenciar a partir de la relación $\mathrm{dv} / \mathrm{d}$; para $C$. pipiens esta relación va de 0 a 0,5 , mientras que para $C$. quinquefasciatus está entre 0,5 y 1,4. En un estudio llevado a cabo por Humeres et al. en 1998 (9) en la región central de Argentina, donde hay intergradación de las dos especies, los autores, basados en la divergencia y flujo genético, sugieren nuevamente que son dos subespecies ya que el entrecruzamiento de $C$. pipiens y $C$. quinquefasciatus es estable y ocurre en los sitios en donde la temperatura es favorable para el crecimiento de las dos (7).

C. quinquefasciatus es considerada una especie acentuadamente antropofílica (12) y es el mosquito del género Culex que se encuentra asociado con mayor frecuencia al hábitat humano tanto urbano como rural en Colombia. Esta especie se ha relacionado con la transmisión de filarias como Wuchereria bancroftiy Dirofilaria immitis, del virus del Nilo occidental y de los virus causantes de la encefalitis de San Luis y la encefalitis equina venezolana, entre otros $(2,13)$. En áreas donde no existe riesgo de transmisión de agentes patógenos por parte de esta especie, constituye un problema de salud pública debido a la alergia ocasionada por su picadura y a las molestias causadas por las altas densidades de población que alcanzan, como ocurre en el municipio de Sibaté en la sabana de Bogotá (14).

Los estadios inmaduros se desarrollan preferiblemente en depósitos de agua y los criaderos son variados, constituidos por aguas con un alto grado de contaminación, abundante contenido de materia orgánica, con detritos en proceso de fermentación, en ambientes sombreados, lénticos o semilóticos, cercanos al ambiente domiciliario (6).

Debido a su antropofilia acentuada, la resistencia a los insecticidas, su capacidad vectorial y a las altas densidades que puede llegar a alcanzar, $C$. quinquefasciatus es un factor de riesgo para las poblaciones urbanas (14-17). Por tanto, el conocimiento de los aspectos biológicos, 
fisiológicos y bioquímicos de la especie se constituyen en el primer paso que conlleva a la implementación de medidas que conduzcan al control de las poblaciones de mosquitos en las áreas urbanas.

En este estudio se determinó la duración del desarrollo de las etapas inmaduras del mosquito C. quinquefasciatus proveniente de la sabana de Bogotá en condiciones de laboratorio, mediante dos experimentos realizados durante el 2001.

\section{Metodología}

\section{Captura y mantenimiento de los mosquitos}

Los mosquitos adultos fueron capturados en la finca Marengo localizada a $4^{\circ} 42^{\prime}$ latitud norte y $74^{\circ} 14^{\prime}$ longitud oeste a $2.543 \mathrm{msnm}$, en inmediaciones de la sabana de Bogotá, municipio de Mosquera (Cundinamarca) y se transportaron hasta el Laboratorio de Parasitología de la Facultad de Medicina de la Universidad Nacional para establecer el criadero y para su identificación. Los parentales fueron transferidos a una jaula $(1 \mathrm{~m} \mathrm{x}$ $1 \mathrm{~m} \times 1 \mathrm{~m})$ y se mantuvieron con una dieta de sacarosa al $10 \%$. Como fuente sanguínea para las hembras, se introdujo en la jaula un ratón inmovilizado, previamente anestesiado según el protocolo del Bioterio Central de la Universidad Nacional de Colombia, el cual se acoge a la Ley 84 de 1989. Se dispusieron recipientes de barro color natural y pintados de negro, y recipientes plásticos transparentes y blancos, a los cuales se les agregó agua de diferentes procedencias: charca, grifo y agua destilada, para que la hembra depositara sus huevos. Las balsas de huevos (se transfirieron a un recipiente con agua destilada y se transportaron a otra jaula $(50 \mathrm{~cm} \times 50 \mathrm{~cm} \times 50$ $\mathrm{cm}$ ) en donde las larvas se alimentaron con concentrado para perros. El criadero se mantuvo bajo condiciones no controladas de luz, temperatura y humedad relativa durante todo el experimento. Las ventanas del recinto se mantuvieron cerradas para evitar el escape de los mosquitos lo que, a su vez, evitaba mayores variaciones climáticas en el criadero.

\section{Ciclo de vida}

Se seleccionaron aleatoriamente 4 balsas de huevos que fueron examinadas bajo estereoscopio para determinar el número de huevos en cada una de ellas y se midió la longitud y el ancho. Posteriormente, se escogieron otras 4 balsas de un tamaño similar a las observadas y se depositaron individualmente en recipientes con agua destilada, los cuales se revisaron diariamente. A medida que las larvas emergían o cambiaban de estadio, se trasladaron a recipientes rotulados con la fecha y el número de larvas depositadas. De igual manera, cuando las larvas mudaron a pupas se trasladaron a nuevos recipientes debidamente identificados con el fin de establecer el número de pupas y la duración del estadio de pupa. Para establecer el número de adultos, se contabilizó el número de exuvias pupales que quedaron en los recipientes. Se registró el número de individuos que pasó exitosamente de un estadio a otro y los que no lo lograron.

Los datos se utilizaron para hacer una tabla de vida vertical, donde, $x=$ etapa de desarrollo; $I_{x}=$ proporción de sobrevivientes en la etapa $x\left(\mathrm{~N}_{x} /\right.$ $\mathrm{N}_{\mathrm{o}}$ ); $\mathrm{d}_{\mathrm{x}}=$ número de individuos que mueren entre las etapas $(x-1)$ y $x\left(N_{x-1}-N_{x}\right) ; q_{x}=$ probabilidad de morir entre $x-1$ y $x\left(d_{x} / N_{x-1}\right)$ y $L_{x}=$ media de la probabilidad de supervivencia entre $\mathrm{x}-1$ y $\mathrm{x}\left(\mathrm{I}_{\mathrm{x}}+\right.$ $\left.\mathrm{I}_{x+1}\right) / 2$ (18). También se consignaron el porcentaje de eclosión, el porcentaje de huevos que pasan a larvas LI; el de pupación, porcentaje de larvas L1 que culminan exitosamente la pupación y el de emergencia, porcentaje de pupas que terminan su desarrollo e inician la vida adulta.

Para determinar la duración del desarrollo de las etapas inmaduras de C. quinquefasciatus se llevaron a cabo dos experimentos, el primero durante el periodo seco (28 de enero-28 de febrero) y el segundo durante la época de lluvia (13 de septiembre-15 de octubre) de 2001. Los datos de temperatura media y humedad relativa diarias fueron registrados en la Estación Meteorológica de la Universidad Nacional durante los dos periodos del experimento.

\section{Análisis estadístico}

Los datos se analizaron mediante el programa estadístico SPSS 7.5. Se realizó la prueba F para homogeneidad de varianzas entre los experimentos realizados en las dos épocas diferentes del año (18-20). 


\section{Resultados}

La identificación de los ejemplares se realizó mediante el uso de las descripciones y claves $(11,12)$. La relación dv/d media encontrada en los ejemplares fue de 0,67 , relación que como ya ha sido descrita por varios autores corresponde a $C$. quinquefasciatus $(10-11,21,22)$ (figura 1).

El día inicial del ciclo correspondió al momento en el que las hembras ingirieron sangre y el día final fue aquel en el cual emergió el último adulto. Entre los dos experimentos no hubo diferencia estadísticamente significativa $(p>0,05)$ en la duración de cada una de las etapas. Los mosquitos se mantuvieron en condiciones naturales de luz, temperatura y humedad relativa durante ambos periodos. La temperatura promedio durante el

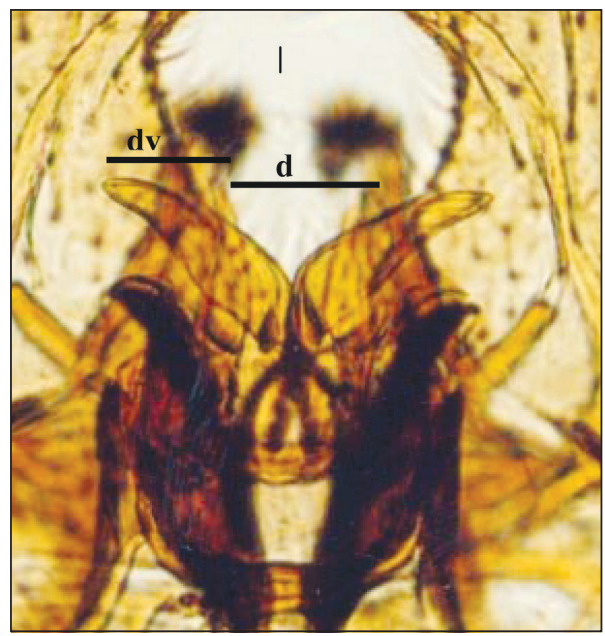

Figura 1. Terminalia del macho; se observan las distancias entre los brazos ventrales y dorsales del mesosoma. Convenciones: dv: distancia entre los brazos ventrales y dorsales; d: distancia entre los brazos dorsales. primer periodo fue de $14,8^{\circ} \mathrm{C}$ y de $15,1^{\circ} \mathrm{C}$ durante el segundo, mientras que la humedad relativa promedio fue $72,5 \%$ y $74,1 \%$, respectivamente.

En el cuadro 1 se consignan los datos correspondientes a la duración promedio de cada etapa de desarrollo para los experimentos bajo las condiciones enunciadas anteriormente. Para la oviposición, las hembras mostraron una preferencia marcada por los recipientes de plástico transparente que contenían agua de charca. El tiempo que tardaron las hembras en depositar los huevos, una vez habían ingerido sangre, varió entre 5 y 8 días; la mayor variación en cuanto a la duración de cada etapa se presentó en los estadios larvarios III y IV. La duración mínima del ciclo entre el periodo de huevo a adulto fue de 13 días y la máxima de 24 días. La etapa de desarrollo que presentó menor duración fue la de pupa, con un $11 \%$ del tiempo de desarrollo total; el tercer y el cuarto estadio larvarios tuvieron la duración promedio mayor $(18 \%$ y $29 \%$ del tiempo de desarrollo total, respectivamente), mientras que las otras etapas tuvieron la misma duración, cada una con el $14 \%$.

En el cuadro 2 se consignan los resultados de supervivencia y mortalidad para cada estadio de desarrollo en las condiciones estudiadas y los datos concernientes a los porcentajes de eclosión, pupación y emergencia para $C$. quinquefasciatus. En el primer experimento sólo emergieron larvas a partir de dos balsas de huevos (50\%), mientras que en el segundo eclosionaron huevos de tres balsas (75\%). El número de huevos por balsa varió entre 152 y 203. En el primer experimento los parámetros para la tabla de vida se empezaron a

Cuadro 1. Duración en días de las etapas de desarrollo de C. quinquefasciatus (E1: experimento 1, enero-febrero de 2001; E2: experimento 2, septiembre-octubre de 2001).

\begin{tabular}{llccccccc}
\hline & & $\begin{array}{c}\text { Ingestión - Huevo } \\
\text { huevo }\end{array}$ & Larva I & Larva II & Larva III & Larva IV & Pupa \\
\cline { 2 - 8 } E1 & $\begin{array}{l}\text { Promedio } \\
\text { (días) }\end{array}$ & 7,0 & 1,5 & 2,2 & 2,2 & 3,0 & 5,0 & 1,5 \\
& IC 95\% & $5,05-8,95$ & $0,52-2,48$ & $2.09-2,31$ & $2,08-2,32$ & $76-3,24$ & $4,75-5,25$ & $1,37-1,63$ \\
E2 & $\begin{array}{l}\text { Promedio } \\
\text { (días) }\end{array}$ & 5,5 & 2,7 & 2,7 & 2,7 & 3,5 & 5,3 & 2,0 \\
IC 95\% & $3,38-7,62$ & $1,9-3,5$ & $1,9-3,5$ & $1,9-3,5$ & $1,38-5,62$ & $3,18-7,42$ & $1,05-2,95$ \\
\hline
\end{tabular}


Cuadro 2. Tabla de vida Culex quinquefasciatus.

\begin{tabular}{|c|c|c|c|c|c|c|}
\hline & Etapa & $\begin{array}{l}\mathrm{N}^{\circ} \text { promedio } \\
\text { contabilizado }\end{array}$ & $\mathrm{I}_{\mathrm{x}}{ }^{1}$ & $d_{x}^{2}$ & $q_{x}^{3}$ & $\mathrm{~L}_{x}^{4}$ \\
\hline $\begin{array}{l}\text { Experimento } 1 \\
\text { (enero a } \\
\text { febrero de 2001) }\end{array}$ & $\begin{array}{l}\text { Huevo } \\
\text { Larva I } \\
\text { Larva II } \\
\text { Larva III } \\
\text { Larva IV } \\
\text { Pupa } \\
\text { Adulto }\end{array}$ & $\begin{array}{l}152 \\
139 \\
131 \\
127 \\
124\end{array}$ & $\begin{array}{l}0,9174 \\
0,9424 \\
0,9694 \\
0,9724\end{array}$ & $\begin{array}{c}13 \\
8 \\
4 \\
4\end{array}$ & $\begin{array}{l}0,0825 \\
0,0575 \\
0,0305 \\
0,0275\end{array}$ & $\begin{array}{l}0,9299 \\
0,9559 \\
0,9709\end{array}$ \\
\hline $\begin{array}{l}\text { Experimento } 2 \\
\text { (septiembre a } \\
\text { octubre de 2001) }\end{array}$ & $\begin{array}{l}\text { Huevo } \\
\text { Larva I } \\
\text { Larva II } \\
\text { Larva III } \\
\text { Larva IV } \\
\text { Pupa } \\
\text { Adulto } \\
\text { Eclosión } \\
\text { Pupación (larva-pupa) } \\
\text { Emergencia (pupa-adulto) }\end{array}$ & $\begin{array}{l}203 \\
170 \\
167 \\
165 \\
155 \\
153 \\
152\end{array}$ & $\begin{array}{l}0,8357 \\
0,9823 \\
0,9920 \\
0,9375 \\
0,9870 \\
0,9956\end{array}$ & $\begin{array}{c}33 \\
3 \\
1 \\
10 \\
2 \\
1 \\
76,1-91,1 \% \\
79,1-92,9 \% \\
91,1-99,9 \%\end{array}$ & $\begin{array}{l}0,1642 \\
0,0176 \\
0,0080 \\
0,0625 \\
0,0129 \\
0,0043\end{array}$ & $\begin{array}{l}0,9090 \\
0,9871 \\
0,9647 \\
0,9622 \\
0,9913\end{array}$ \\
\hline
\end{tabular}

$1_{x}:$ proporción de sobrevivientes en la etapa $x$

${ }^{2} d_{x}$ : número de individuos que mueren entre las etapas $(x-1)$ y $x$

${ }^{3} \mathrm{q}_{\mathrm{x}}$ : probabilidad de morir entre $(\mathrm{x}-1)$ y $\mathrm{x}\left(\mathrm{d}_{\mathrm{x}} / \mathrm{N}_{\mathrm{x}-1}-\mathrm{N}_{\mathrm{x}}\right)$

${ }^{4} \mathrm{I}_{\mathrm{x}}$ : media de la probabilidad de supervivencia entre $(x-1)$ y $x\left(\mathrm{I}_{\mathrm{x}}+\mathrm{I}_{\mathrm{x}+1}\right) / 2$

considerar desde el segundo estadio larval, mientras que en el segundo se inició desde el momento mismo de la oviposición. No se encontró diferencia significativa entre los experimentos para las variables proporción de supervivientes $\left(I_{x}\right)$ ( $f=5,81, F=9,01$; alfa $=0,05)$, probabilidad de morir entre dos etapas $\left(q_{x}\right)(f=5,81, F=9,01$; alfa=0,05) y probabilidad media de supervivencia entre dos etapas $\left(L_{x}\right)(f=2,5 ; F=19,25 ;$ alfa $=0,05)$. Aunque la proporción de supervivientes fue alta en todas las etapas, la mayor probabilidad de morir se presentó durante el paso de huevo a larva $(0,1642)$; a partir de este momento, la mortalidad se redujo considerablemente.

\section{Discusión}

La amplia distribución de C. quinquefasciatus tanto en el hemisferio norte como en el sur expone a esta especie a una variedad de climas y condiciones que son un reto para su supervivencia. En Colombia, C. quinquefasciatus se distribuye por casi todo el territorio nacional en alturas que van desde los 0 hasta los 3000 msnm (Becerra J. Proyecto: Estudio de los zancudos picadores que se encuentran en el
Distrito Capital de Santafé de Bogotá. Secretaría Distrital de Salud, Subdirección de Vigilancia Epidemiológica. Santafé de Bogotá, 1992). Muchos de los estudios acerca del ciclo de vida de esta especie, se han realizado bajo condiciones controladas en laboratorio (22-24). Por el contrario, los datos presentados en este estudio ofrecen una idea aproximada de la dinámica de poblaciones en esta especie de mosquito en Bogotá, puesto que los individuos a los cuales se les hizo el seguimiento se mantuvieron bajo condiciones no controladas de luz, temperatura y humedad relativa.

En su ambiente natural, las larvas se desarrollan en aguas contaminadas, ricas en materia orgánica y, al parecer, tal preferencia está condicionada por estímulos de naturaleza química y olfatoria (24). Tal comportamiento también fue observado en este caso, ya que las hembras prefirieron realizar la oviposición en recipientes de plástico con agua de charca con alto contenido de materia orgánica y evitaban aquéllos que contenían agua de grifo o agua destilada. Los recipientes de barro que les proporcionan ambientes sombreados 
también pueden ser utilizados con buenos resultados, ya que esta especie muestra acentuada preferencia por sitios oscuros para reposar (25).

La temperatura media ambiental durante los dos experimentos fue de $14,8^{\circ} \mathrm{C}$ y $15,1^{\circ} \mathrm{C}$, respectivamente y no hubo diferencia significativa entre los ensayos realizados en diferentes épocas del año. Cabe anotar, sin embargo, que la duración media de cada etapa fue ligeramente superior durante el segundo experimento (septiembre-octubre), lapso en el que también se presentaron valores de temperatura media y humedad relativa un poco mayores. Los tiempos de desarrollo estimados para $C$. quinquefasciatus en este estudio fueron más cortos que los reportados para esta especie por Rueda et al. (22) para una temperatura constante de $15^{\circ} \mathrm{C}$; estos mismos autores encontraron que el número de días requerido para completar el desarrollo de huevo a adulto en un rango de temperaturas entre $15^{\circ} \mathrm{C}$ y $27^{\circ} \mathrm{C}$ es menor que a temperaturas mayores. Oda et al. (24) reportaron que no hubo un descenso en la actividad reproductora o en la tasa de supervivencia de $C$. quinquefasciatus en un rango de temperaturas entre $21^{\circ} \mathrm{C}$ y $30^{\circ} \mathrm{C}$, lo que demuestra la gran capacidad de adaptación de esta especie a diversas condiciones.

Oda et al. (24) reportaron que una de las diferencias fisiológicas entre $C$. pipiens y $C$. quinquefasciatus de Japón era la incapacidad de esta última especie de pasar por periodos de diapausa. Sin embargo, en el criadero del laboratorio se ha observado la resistencia de los huevos de $C$. quinquefasciatus a la desecación y se ha producido la eclosión de larvas, incluso un mes después de la ovipostura, cuando los huevos se humedecen nuevamente.

Los porcentajes de eclosión de las balsas a partir de las cuales emergieron larvas, al igual que los porcentajes de pupación y emergencia de adultos fueron altos; todos estos parámetros estuvieron por encima del $80 \%$; se destaca que el porcentaje de emergencia fue cercano al $100 \%$ pero no fue sincrónico, lo que quiere decir que no emergieron larvas de todas las balsas que se estaban observando y que el índice de supervivencia fue muy alto en las últimas etapas del desarrollo larval Esto sugiere que las etapas más críticas en el ciclo de vida se ubican en la etapa de huevo y los dos primeros estadios larvales que son justamente en los cuales estos individuos pasan el menor porcentaje de tiempo de su desarrollo.

Teniendo en cuenta que los esfuerzos dirigidos al control de las poblaciones de mosquitos se han enfocado principalmente a la aplicación de insecticidas y que $C$. quinquefasciatus ha desarrollado resistencia múltiple rápidamente (1517) se ha venido implementando el control biológico de las etapas larvales por medio de nemátodos como Romanomernis culicivorax o bacterias como Bacillus sphaericus (23). Según los datos de este estudio, la etapa más vulnerable es la de huevo porque la eclosión a L1 estuvo entre $50 \%$ y $75 \%$, pero debido a la naturaleza asincrónica de eclosión este porcentaje puede variar al hacer un seguimiento más prolongado a las balsas en las que no se observó eclosión durante el período del estudio, a diferencia de los porcentajes de supervivencia en las demás etapas que son superiores al $80 \%$.

Los resultados indican la gran adaptación de $C$. quinquefasciatus a las condiciones establecidas en la sabana de Bogotá que se ve favorecida, además, por las alteraciones producidas por el hombre en el ambiente a medida que establece nuevos asentamientos fomentando la aparición de nuevos criaderos o la eutroficación de las fuentes de agua existentes. Además, la rapidez con la que $C$. quinquefasciatus alcanza el estado adulto es una característica importante de esta especie, ya que aumenta la capacidad vectorial de este mosquito.

Aunque en el área donde fueron capturados los parentales no existen estudios de resistencia, el hecho de que tal fenómeno se haya reportado para organofosforados en cepas colombianas (26), la resistencia al control biológico con $B$. thuringiensis en otras partes del mundo (27), aunados al corto periodo en el que se completa el ciclo de vida de C. quinquefasciatus, pueden hacer pensar que las medidas de control deben ser integrales utilizando control físico, químico y biológico y de carácter permanente. Por el rápido desarrollo del ciclo de 
vida, las medidas deben tener un alto impacto en la población de mosquitos igualmente en un lapso de tiempo muy corto para lograr la reducción de las densidades de población a niveles en que no constituyan problema para las poblaciones humanas. Para el caso de la cepa estudiada y con el fin de tener más herramientas para su control, se deben hacer estudios de resistencia a insecticidas químicos y biológicos.

\section{Referencias}

1. Travi B, Montoya J. Manual de entomología médica para investigadores de América Latina. Cali, Colombia: Cideim; 1994. p.90-142.

2. Rivas F, Díaz L, Cárdenas V, Daza E, Bruzón L, Alcalá A et al. Epidemic Venezuelan equine encephalitis in La Guajira, Colombia, 1995. J Infect Dis 1997;175:82832.

3. Sabatinelli G, Ranieri E, Gianzi FP, Papakai M, Cancrini G. Role de Culex quinquefasciatus dans la transmisión de la filariose de Bancroft dans la République Fedérale Islamique des Comores (Océan Indien). Parasite 1994;1:71-6.

4. Weaver S, Salas R, Rico-Hesse R, Ludwig G, Oberste S, Boshell J et al. Re-emergence of epidemic Venezuelan equine encephalomyelitis in South America. Lancet 1996;348:436-40.

5. Savage H, Miller B. House mosquitoes of the U.S.A., Culex pipiens complex. Win Beats 1995;6:8-9.

6. Brewer M, Bufa L, Almirón W. Culex pipiens quinquefasciatus y Culex pipiens pipiens (Diptera: Culicidae) en Córdoba, Argentina. Rev Per Entomol 1987; 29:69-72.

7. Almirón WR, Humeres SG, Gardenal SN. Distribution and hybridization between Culex pipiens and Culex quinquefasciatus (Diptera: Culicidae) in Argentina. Mem Inst Oswaldo Cruz 1995;90:469-73.

8. Forattini OP. Entomología médica. Culicini: Culex, Aedes e Psorophora. Sao Paulo: Editora da Universidade de Sao Paulo; 1965.

9. Humeres S, Almirón W, Sabattini M, Gardenal C. Estimation of genetic divergence and gene flow between Culex pipiens and Culex quinquefasciatus (Diptera: Culicidae) in Argentina. Mem Inst Oswaldo Cruz 1998; 93:57-62.

10. Peng Z, Simons E. Cross-reactivity of skin and serum specific IgE responses and allergen analysis for three mosquito species with worldwide distribution. J Allergy Clin Immunol 1997;100:192-8.

11. Jupp PG. Culex (Culex) pipiens pipiens Linnaeus and Culex (Culex) pipiens quinquefasciatus Say in South Africa: morphological and reproductive evidence in favor of their status as two species. Mosq Sys 1978; 10:461-73.

12. Forattini O, Kakitani I, La Corte Dos Santos R, Kobayashi K, Ueno H, Fernández Z. Potencial sinantrópico de mosquitos Kertesia e Culex (Diptera: Culicidae) no Sudeste do Brasil. Rev Saúde Pública 2000;34:565-9.

13. Goddard LB, Roth AE, Reisen WK, Scott TW. Vector competence of California mosquitoes for West Nile Virus. Em Infect Dis 2002;8:1385-91.

14. Sarmiento MJ, Idrovo J, Restrepo M, Díaz MP, González A. Evaluación del impacto de la contaminación del embalse del Muña sobre la salud humana. Revista de Salud Pública 1999;1:159-71.

15. Bisset J, D’Eguez L, Rodríguez M, Díaz C, González T, Vázquez R. Tres combinaciones de esterasas y su relación con la resistencia a insecticidas organofosforados, carbamatos y piretroides en Culex quinquefasciatus Say, 1823 (Diptera: Culicidae) de Cuba. Rev Cub Med Trop 1996;48:5-11.

16. Desilva D, Hemingway J, Ramson H, Vaughan A. Resistance to insecticides in Insect vectors of disease: est-a3, a novel amplified esterase associated with amplified est-b1 from insecticide resistant strains of the mosquito Culex quinquefasciatus. Exp Parasitol 1997;87:253-9.

17. Meisch M, Meek C, Brown J, Núñez R. Field trial efficacy of two formulations of Permanone against Culex quinquefasciatus and Anopheles quadrimaculatus. J Am Mosq Control Assoc 1997;13:311-4.

18. Rabinovich J. Ecología de poblaciones animales. Washington, D.C.: OEA; 1978.

19. Cárdenas E, Ferro C, Corredor D, Martínez O, Munstermann L. Reproductive biology of Lutzomyia shanonni (Dyar) (Diptera: Psychodidae) under experimental conditions. J Vector Ecology 1999;24:158-70.

20. Snedecor GW, Cochran WG. Statistical methods. Sixth edition. Ames, lowa: lowa State University Press; 1987. p.56-61.

21. La Casse WJ, Yamaguti S. Mosquito fauna of Japan and Korea. Washington, D.C.: USAF School of Aviation Medicine Air University; 1950.

22. Rueda M, Patel K, Axtell R, Stinner E. Temperaturedependent development and survival rates of Culex quinquefasciatus and Aedes aegypti (Diptera: Culicidae). J Med Entomol 1990;27:892-6.

23. Jittawadee R, Mulla M. Biological fitness of Culex quinquefasciatus (Diptera: Culicidae) susceptible and resistant to Bacillus sphaericus. J Med Entomol 1997;34: 5-9.

24. Oda T, Uchida K, Mori A, Mine M, Eshita Y, Kurokawa $\mathbf{K}$ et al. Effects of high temperature on the emergence and survival of adult Culex pipiens molestus and Culex 
quinquefasciatus in Japan. J Am Mosq Control Assoc 1999;15:153-6.

25. Wen Y, Muir L, Kay B. Response of Culex quinquefasciatus to visual stimuli. J Am Mosq Control Assoc 1997;13:150-2.

26. Bisset J, Rodríguez MM, Díaz C, Soca A. Estudio de la resistencia de una cepa de Culex quinquefasciatus procedente de Medellín. Rev Cubana Med Trop 1998;50: 133-7.

27. Rahman M, Roberts H, Sarjan M, Asgari S, Schmidt O. Induction and transmission of Bacillus thuringiensis tolerance in the flour moth Ephestia kuehniella. PNAS 2004;101:2696-9. 